\title{
Talin1 knockdown prohibits the proliferation and migration of colorectal cancer cells via the EMT signaling pathway
}

\author{
LING JI $^{1,2}$, FEIZHAO JIANG ${ }^{2}$, XIANPING CUI ${ }^{1}$ and CHENGKUN QIN ${ }^{1}$ \\ ${ }^{1}$ Department of Hepatobiliary Surgery, Shandong Provincial Hospital Affiliated to Shandong University, \\ Jinan, Shandong 250021; ${ }^{2}$ Department of Colorectal Surgery, The First Affiliated Hospital of \\ Wenzhou Medical University, Wenzhou, Zhejiang 325000, P.R. China
}

Received November 29, 2018; Accepted August 6, 2019

DOI: $10.3892 / \mathrm{ol} .2019 .10902$

\begin{abstract}
Colorectal cancer (CRC) is the third most commonly diagnosed cancer and the second highest cause of cancer-associated death worldwide. Talin1 activates integrins, which mediate cell adhesion, proliferation, tumorigenesis and metastasis. The aim of the present study was to determine talin1 expression levels in colorectal cancer (CRC) and investigate the role of talin1 in CRC proliferation and invasion in vitro and in vivo. Talin1 protein expression levels were detected in human CRC and adjacent normal tissues by immunohistochemistry. Talin1 short hairpin RNA and control vectors were designed and stably transfected into HCT116 CRC cells. Cell proliferation was determined by MTT assay. Cell migratory and invasive capabilities were detected by wound-healing and Matrigel invasion assays. The expression of proteins in the epithelial-to-mesenchymal transition signaling pathway was determined by western blotting and reverse transcription-quantitative PCR. The effect of talin1 on tumor growth was explored in vivo using $\mathrm{BALB} / \mathrm{c}$ nude mice. Immunohistochemical analysis of CRC and adjacent normal tissue revealed that talin1 expression was upregulated in CRC. Talin 1 knockdown significantly reduced the proliferation, migration and invasive ability of HCT116 cells compared with the control. Protein levels of phosphorylated STAT3 and vimentin were significantly lower in talin1-knockdown HCT116 cell lines compared with the control, whereas protein levels of E-cadherin were increased. Interleukin-6 mRNA levels were significantly increased in patients' blood samples compared with blood samples from healthy controls, as well as in CRC compared with adjacent normal tissue. In vivo experiments demonstrated that talin1
\end{abstract}

Correspondence to: Professor Chengkun Qin, Department of Hepatobiliary Surgery, Shandong Provincial Hospital Affiliated to Shandong University, 324 Jingwuwei 7th Road, Jinan, Shandong 250021, P.R. China

E-mail: qinchengkun_u_sly@163.com

Key words: talin1, colorectal cancer, epithelial-to-mesenchymal transition, proliferation, migration knockdown reduced CRC tumor growth and weight in nude mice. In conclusion, Talin 1 knockdown may prevent the proliferation and migration of CRC cells by downregulating various factors involved in the epithelial-to-mesenchymal transition process, such as phosphorylated STAT3 and vimentin; therefore, talin1 may provide a novel therapeutic target for CRC.

\section{Introduction}

Globally, colorectal cancer (CRC) is a leading cause of cancer-related mortality (1). In a number of low- and medium-income countries, CRC incidence and mortality rates are increasing (2). The incidence and mortality rates are 1.1 and $0.6 \%$, respectively, in less developed areas, lower than the 2.7 and $1 \%$ incidence and mortality rate of developed countries (2), The difference is rates between developed and underdeveloped countries may be attributable to the adoption of Western diets and sedentary lifestyles (3). In the UK, an estimated $50 \%$ of patients with CRC develop metastasis, and the 5-year survival rate for all CRC patients in the UK is approximately $55 \%$ (4). As such, there is an urgent need to identify the molecular mechanisms underlying the pathophysiology of CRC and develop potential therapeutic targets.

Talin1 is a macromolecular $(270 \mathrm{kDa})$ adaptor protein localized in adhesion complexes between cells and the extracellular matrix (ECM). Talin1 binds to and activates integrins, directly connecting integrins to the actin cytoskeleton and recruiting numerous proteins, such as vinculin and focal adhesion kinase (FAK), into integrin adhesion complexes (IACs) (5-7). IACs provide adhesive structures between cells and the ECM and control cell survival, proliferation, invasion and migration $(8,9)$. Talin1 may also regulate cadherin-mediated cell adhesion in an integrin-independent manner (10).

Previous studies have demonstrated that talin 1 is expressed in several human cancers, including liver (11) and prostate (12) cancer and oral squamous cell carcinoma (13), and that talin1 serves a role in tumor formation, migration and metastasis. Specifically, genes regulated by talin1 may be involved in epithelial-to-mesenchymal transition (EMT) (14). EMT is characterized by a temporary reversible shift to a mesenchymal phenotype in cancer cells, leading to cancer progression and metastasis (15). 
The STAT3 signaling pathway regulates EMT, which contributes to cancer progression (16). STAT3 is activated in thymic epithelial tumors (17) and colorectal adenocarcinoma (18), whereas inhibition of the STAT3 signaling pathway represses growth and invasion and induces apoptosis in a number of different types of tumors, including pancreatic cancer, prostatic carcinomas, and head and neck tumors (19-21).

Inflammation contributes to the pathophysiology of cancer, including tumor initiation, promotion, progression and metastasis, and is involved in the immune surveillance of early cancer and response to therapy $(22,23)$. Interleukin- 6 (IL-6) exhibits pro-tumorigenic activities, affecting tumor cell proliferation, survival, differentiation, migration, invasion and metastasis, as well as serving a role in angiogenesis, inflammation and metabolism (24). IL-6 exerts pro-angiogenic activity predominantly through STAT3 signaling, which leads to the induction of hypoxia-inducible factor-1-mediated vascular endothelial growth factor A transcription, as well as endothelial cell proliferation and migration (25-27).

Currently, studies focusing on the expression levels and role of talin 1 in CRC are limited. The aims of the present study were to determine the expression of talin1 by immunohistochemistry (IHC) in human CRC samples and to explore the role of talin1 in CRC using short hairpin (sh)RNA knockdown in HCT116 and mouse xenograft models.

\section{Materials and methods}

Patients and samples. CRC, adjacent normal tissues and whole blood samples were obtained from 30 patients (mean age, 53 years; range, 36-73 years; 19 males and 11 females) admitted for surgical treatment in the Department of General Surgery, The First Affiliated Hospital of Wenzhou Medical University (Wenzhou, China) between January 2016 and June 2017. Each patient had an available specimen of resected primary CRC. All patients with CRC were classified according to the TNM staging system using the International Union Against Cancer criteria (28). Patients who were diagnosed with a cancer of any other histotype and those with a family history of CRC were excluded from the study. Pathological classification of tumors was assessed using the World Health Organization criteria (28). CRC and adjacent normal tissue samples were sectioned into two parts; One part of the tissue sample was fixed with $4 \%$ paraformaldehyde for $24 \mathrm{~h}$ at $4^{\circ} \mathrm{C}$, subsequently embedded in paraffin and used for histopathological examination, whereas the other part was flash-frozen in liquid nitrogen and stored at $-80^{\circ} \mathrm{C}$ until further use. Whole blood was collected in PAXgene Blood RNA tubes (Qiagen GmbH). In July 2016, control blood samples were collected from 24 healthy donors and laboratory staff at the Department of General Surgery, The First Affiliated Hospital of Wenzhou Medical University (mean age, 51 years; range, 39-69 years; 10 males and 14 females) who had no history of malignancy, inflammatory or infectious diseases. This study was approved by the Board of the Ethical Committee of The First Affiliated Hospital of Wenzhou Medical University. Written informed consent in accordance with the Declaration of Helsinki was obtained from all study subjects.
Cell culture. HCT116 cells and 293T cells (American Type Culture Collection) were cultured in DMEM with $10 \%$ fetal bovine serum (FBS; Invitrogen, Thermo Fisher Scientific, Inc.), penicillin G (100 U/ml; Sangon Biotech Co., Ltd.) and streptomycin (100 mg/ml; Sangon Biotech Co., Ltd.) at $37^{\circ} \mathrm{C}$ in a humidified atmosphere containing $5 \% \mathrm{CO}_{2}$.

Preparation of viruses and cell infection. VSV-G-pseudotyped lentiviral vectors were produced by co-transfecting $6 \times 10^{6}$ 293T cells with 2 pmol scrambled short hairpin (sh)RNA as a control talin1 1\# TRCN0000123105, 5'-CCGGGCAGTGAA AGATGTAGCCAAACTCGAGTTTGGCTACATCTTTCA CTGCTTTTTG-3' or talin1 2\# TRCN0000299020, 5'-CCG GGCCTCAGATAATCTGGTGAAACTCGAGTTTCACCA GATTATCTGAGGCTTTTTG-3' shRNA (Sigma-Aldrich; Merck KGaA) using Lipofectamine ${ }^{\circledR} 2000$ (Invitrogen; Thermo Fisher Scientific, Inc.) and the following plasmids (all from Invitrogen; Thermo Fisher Scientific, Inc.): 1 pmol pMDLg/RRE, 0.5 pmol pMD2.G and $0.5 \mathrm{pmol} \mathrm{pRSV-Rev}$, according to the manufacturer's protocol. The culture medium was changed after $8 \mathrm{~h}$. Lentiviral supernatant was collected and filtered through a cellulose acetate membrane $(0.45-\mu \mathrm{m}$ pore) at 48 and $72 \mathrm{~h}$ post-transfection.

HCT116 cells were plated in a 6 well plate $\left(1 \times 10^{5}\right.$ cells/well $)$ and infected with $100 \mu \mathrm{l}$ lentivirus (multiplicity of infection, 20) for $24 \mathrm{~h}$ at $37^{\circ} \mathrm{C}$. Infected cells that stably expressed lentiviral shRNAs were selected with $1 \mathrm{mg} / \mathrm{ml}$ puromycin for 3-4 weeks.

Cell viability. 3-(4,5-Dimethylthiazol-2-yl)-2,5-diphenyltetrazolium bromide (MTT) assays were performed according to a protocol adapted from Mosmann (29). Briefly, talin1-knockdown or shRNA control-transfected HCT116 cells were plated in 96 -well plates at $6 \times 10^{3}$ cells/well and maintained in an incubator at $37^{\circ} \mathrm{C}$ with $5 \% \mathrm{CO}_{2}$ for $\leq 4$ days. Cells in each well were treated with $20 \mu \mathrm{l}$ MTT solution $\left(5 \mathrm{mg} / \mathrm{ml}\right.$ ) for $3 \mathrm{~h}$ at $37^{\circ} \mathrm{C}$. The medium was removed, and cells were lysed with $100 \mu \mathrm{l} \mathrm{DMSO}$ for $2 \mathrm{~h}$ at $37^{\circ} \mathrm{C}$. Cell viability was measured at $570 \mathrm{~nm}$ using an ELISA plate reader (BioTek Instruments, Inc.). Data from three independent experiments were analyzed.

Cell migration assay. Cell migration was assessed using a wound-healing assay. Talin1-knockdown or shRNA control HCT116 cells were cultured to $\sim 100 \%$ confluence in 6-well culture plates. A wound was generated by scratching the monolayer with the tip of a $10-\mu 1$ pipette. The wound was observed using an inverted phase-contrast microscope and digital camera (magnification, $\mathrm{x} 400$ ) at 24 and $48 \mathrm{~h}$. The wound healing rate was calculated as the percentage of the initial wound area that remained at each time point until total wound closure.

Invasion assay. Invasion assay was performed as previously described (30). Briefly, Matrigel-coated (BD Biosciences; $100 \mu 10.35 \mathrm{mg} / \mathrm{ml} ; 1: 30$ dilution in serum-free DMEM) Transwell chambers with $8-\mu \mathrm{m}$ pore size membranes (diameter, $6 \mathrm{~mm}$ ) were incubated at $37^{\circ} \mathrm{C}$ for $6 \mathrm{~h}$. Talin1-knockdown or shRNA control HCT116 cells were trypsinized, washed three times with DMEM containing $1 \%$ FBS, and resuspended in DMEM containing $1 \%$ FBS at a density of $5 \times 10^{5}$ cells $/ \mathrm{ml}$. 
Then, $100 \mu \mathrm{l}$ cell suspension was seeded into upper chambers; $600 \mu$ l DMEM containing 10\% FBS was added to the lower chambers, and chambers were maintained in a $\mathrm{CO}_{2}$ incubator for $12 \mathrm{~h}$. Cells were fixed for $15 \mathrm{~min}$ at room temperature with $3.7 \%$ formaldehyde and stained using $0.1 \%$ crystal violet in $10 \%$ ethanol for $30 \mathrm{~min}$ at room temperature. The number of invaded cells per field in four 4 random fields per well were counted under a light microscope at x200 magnification.

IHC. CRC and adjacent normal tissues were embedded in paraffin. For each patient, the middle part of the CRC and normal paired tissues were punched and transferred to a recipient block to create a tissue microarray (TMA). IHC was performed on $4-\mu \mathrm{m}$ thick sections of paraffin-embedded formalin-fixed samples of tumor and normal tissue from each patient as described previously (31). Briefly, tissue microarray (TMA) slides were baked for $1 \mathrm{~h}$ at $60^{\circ} \mathrm{C}$, deparaffinized, deparaffinized in xylene (three washes, $10 \mathrm{~min}$ each), rehydrated in graded ethanol $(100,95,85$ and $75 \%, 1 \mathrm{~min}$ at each concentration), treated with citrate buffer $\left(\mathrm{pH} \mathrm{6.0)}\right.$ ) at $100^{\circ} \mathrm{C}$ for $30 \mathrm{~min}$, blocked using equine serum at room temperature for $20 \mathrm{~min}$ (Beijing Solarbio Science \& Technology Co., Ltd.) and incubated with $3 \%$ hydrogen peroxide at room temperature for $1 \mathrm{~h}$. TMA slides were incubated with anti-talin1 (1:25 dilution; cat no. ab71333; Abcam) primary antibody at $4^{\circ} \mathrm{C}$ overnight. TMA slides were incubated with a horseradish peroxidase (HRP)-conjugated goat anti-rabbit immunoglobulin G secondary antibody $(1: 2,000$ dilution; cat no. PV-6001; OriGene Technologies, Inc.) at $37^{\circ} \mathrm{C}$ for $1 \mathrm{~h}$, stained using hematoxylin at room temperature for $30 \mathrm{sec}$ (cat no. H8070; Beijing Solarbio Science \& Technology Co., Ltd.), dehydrated using a graded series of ethanol $(50,70,80,90,95$ and $100 \%, 5$ min each) at room temperature, cleared in xylene and mounted on slides using Pertex mounting medium (Pioneer Research Chemicals Ltd.). Talin1 was identified in tissues using ImagePro Plus software 6.0 (Media Cybernetics, Inc.). Using an Eclipse 50i/55i optical microscope at $\mathrm{x} 400$ magnification, the proportion (P) of positive cells was scored as follows: $0,0 \%$ cells stained; $1,1-25 \%$, cells stained; 2,26-50\% cells stained; $3,51-75 \%$ cells stained; and 4, 76-100\% cells stained. All sections were scored by 2 investigators independently, blinded to the origin of the tissue under a light microscope at $\mathrm{x} 400$ magnification. Staining intensity $(I)$ was scored as follows: 0 , no staining; 1 , weak staining; 2 , moderate staining; and 3, strong staining. A histological grade (H-score) was determined according to a previous study as $\mathrm{H}$-score $=\Sigma(I$ x P) (32), and the following expression levels were assigned to each sample: '-', H-score 0-1; '+', H-score 2-3; '++', H-score 4-5; and ' +++ ', $\mathrm{H}$-score $\geq 6$.

Western blot analysis. Talin1-knockdown or control HCT116 cells $2 \times 10^{6}$ were seeded in 6-well plates overnight, washed with ice-cold PBS and harvested using RIPA buffer (Beyotime Institute of Biotechnology). Protein concentration was determined by BCA assay (Beyotime Institute of Biotechnology). Total protein $(20 \mu \mathrm{g})$ was separated using $12 \%$ SDS-PAGE, transferred to a nitrocellulose membrane and blocked with $5 \%$ milk at room temperature for $1 \mathrm{~h}$. Membranes were incubated with primary antibodies against talin1 (1:1,000 dilution), E-cadherin (1:1,000 dilution; cat no. ab15148; Abcam), vimentin (1:1,000 dilution; cat no. ab92547; Abcam), total STAT3 (1:1,000 dilution; cat no. ab119352; Abcam), phosphorylated STAT3 (1:1,000 dilution; cat no. ab76315; Abcam), GADPH (1:1,000 dilution; cat no. ab8245; Abcam) and tubulin (1:1,000 dilution, cat no. ab6046; Abcam) at $4^{\circ} \mathrm{C}$ overnight. Membranes were incubated with a goat anti-rabbit immunoglobulin $\mathrm{G}$ horseradish peroxidase (HRP)-conjugated antibody (1:1,000 dilution; cat. no. A0208; Beyotime Institute of Biotechnology) or goat anti-mouse immunoglobulin G HRP-conjugated antibody (1:1,000 dilution; cat. no. A0216; Beyotime Institute of Biotechnology) at room temperature for $1 \mathrm{~h}$. Color was developed using SuperSignal West Pico PLUS Chemiluminescent substrate (Pierce; Thermo Fisher Scientific, Inc.). GADPH and Tubulin were used as loading controls. Protein levels were quantified using ImageJ software version $1.43 \mathrm{u}$ (National Institutes of Health).

Reverse transcription-quantitative polymerase chain reaction $(R T-q P C R)$. Total RNA was extracted from tumor, normal tissue and blood samples from each patient and talin1-knockdown or shRNA control HCT116 cells using PureLink RNA kit (Ambion; Thermo Fisher Scientific, Inc.) according to the manufacturer's instructions. cDNA was synthesized using 0.5-1.0 $\mu \mathrm{g}$ RNA and the SuperScript First Strand Synthesis kit (Invitrogen; Thermo Fisher Scientific, Inc) at $42^{\circ} \mathrm{C}$ for $50 \mathrm{~min}$ according to the manufacturer's instructions. qPCR was performed using $\mathrm{SYBR}^{\circledR}$ Green PCR Master mix reagents (Invitrogen; Thermo Fisher Scientific, Inc.) on an ABI 7500 Fast Real-Time PCR system (Applied Biosystems; Thermo Fisher Scientific, Inc.), and the thermocycling conditions were as follows: $95^{\circ} \mathrm{C}$ for $20 \mathrm{sec}$, followed by 40 cycles of $95^{\circ} \mathrm{C}$ for $5 \mathrm{sec}$ and $60^{\circ} \mathrm{C}$ for $30 \mathrm{sec}$. The primers used were as follows: Talin1 forward, 5'-TGTTCCCCAGAGCCACCTGCC-3' and reverse, 5'-GAAGCCGCACATCAGGGGC-3'; IL-6 forward, 5'-CAATGAGGAGACTTGCCTGGTG-3' and reverse, 5'-GGTTGGGTCAGGGGTGGTTA-3'; E-cadherin forward, 5'-TGCGCGTGAAGGTTTGCCAGT-3' and reverse, 5'-ACG TTGTCCCGGGTGTCATCCT-3'; occludin forward, 5'-GAC CTTGTCCGTGGATGACTTCAG-3' and reverse, 5'-ATCAGC AGCAGCCATGTACTCTTC-3'; ZO-1 forward, 5'-CACACG ATGCTCAGAGACGAAGG-3' and reverse, 5'-CTGTATGGT GGCTGCTCAAGGTC-3'; and 18S rRNA forward, 5'-ACC TGGTTGATCCTGCCAGT-3' and reverse, 5'-CTGACCGGG TTGGTTTTGAT-3': PCR was performed on an ABI 7500 Realtime PCR system and analyzed using version 2.0.6 of the accompanying software (Applied Biosystems; Thermo Fisher Scientific, Inc.). Results are presented as relative quantification normalized to $18 \mathrm{~S}$ rRNA. The $2^{-\Delta \Delta C q}$ method was used for the relative quantification of gene expression in each sample (33). Each experiment was repeated three times in duplicate.

ELISA. Serum IL-6 concentrations were detected using a Human IL-6 ELISA kit (cat. no. BMS213-2; Invitrogen; Thermo Fisher Scientific, Inc.) according to the manufacturer's instructions. Concentrations of cytokines in standards and samples were measured in triplicate using an iMark microplate reader (Bio-Rad Laboratories, Inc.).

Animal experiments. Animal experiments were approved by the Institutional Animal Committee of Wenzhou Medical University. A total of 20 5-6-week-old BALB/c nude mice (Beijing Vital 


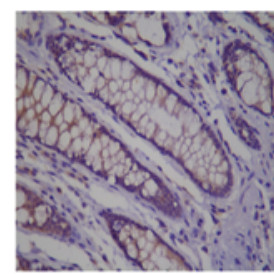

Normal tissue

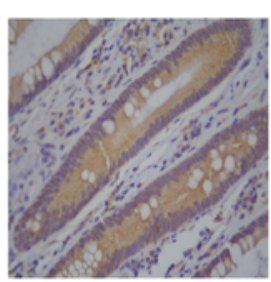

Tumor tissue
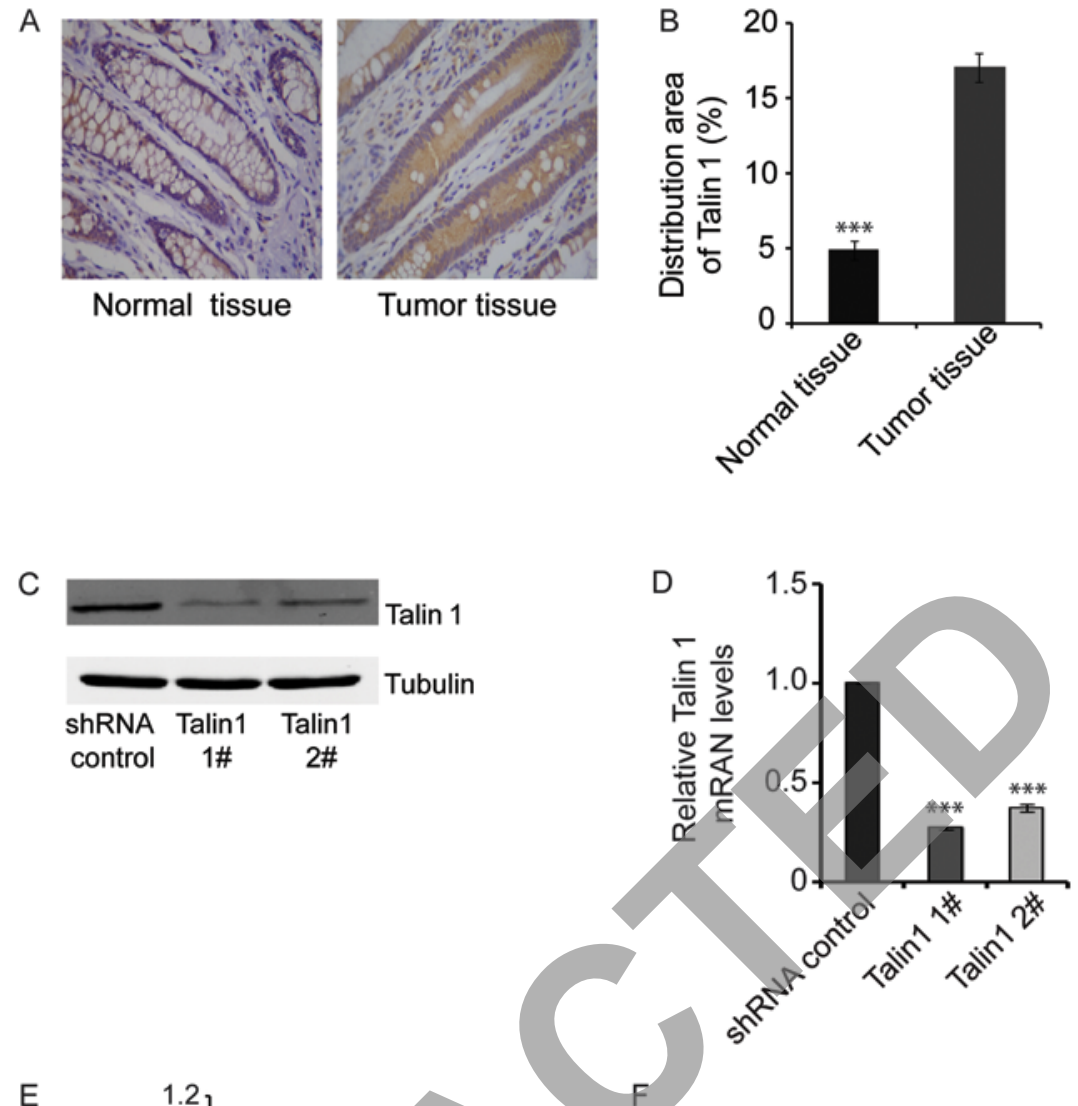

E
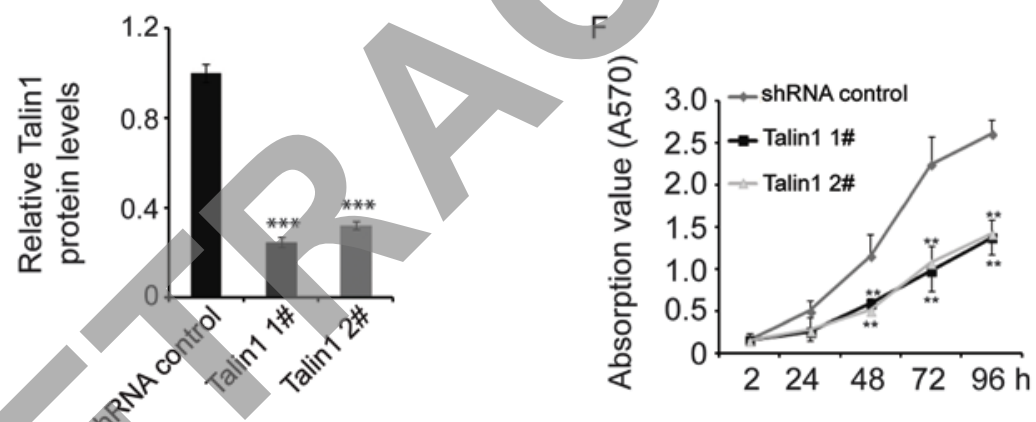

Figure 1. Talin1 expression is upregulated in colorectal cancer and it increases proliferation of HCT116 cells. (A) IHC demonstrating the distribution of talin1 in tumor $(+++)$ and normal (+) tissues. Magnification, x400. (B) Quantification of talin1 distribution in the IHC assays from at least 30 patients. (C) Western blotting results of talin1 protein expression in talin1-knockdown HCT116 cell lines and HCT116 cells transfected with the shRNA control. (D) Talin1 mRNA levels in talin1-knockdown HCT116 cell lines and HCT116 cells transfected with the shRNA control (n=5). (E) Talin1 protein levels in talin1-knockdown HCT116 cell lines and HCT116 cells transfected with the shRNA control $(n=3)$. (F) Knockdown of endogenous talin1 inhibited the proliferation of HCT116 cells. Data are expressed as the mean \pm SEM. ${ }^{* *} \mathrm{P}<0.01$ and ${ }^{* * *} \mathrm{P}<0.001$ vs. control. IHC, immunohistochemistry; shRNA, short hairpin RNA.

River Laboratory Animal Technology Co., Ltd.; 10 per group) were housed at $21-25^{\circ} \mathrm{C}$ with $40-70 \%$ humidity, with a $12 \mathrm{~h}$ dark/light cycle and free access to food and water. A total of $1 \times 10^{6}$ talin1 1\# or shRNA control transfected HCT116 cells were subcutaneously injected. Mouse body weight was measured three times per week. Tumor size was measured every other day with calipers. Tumor volume was calculated using the following formula: Volume $=(\text { width })^{2} \mathrm{x}$ length $/ 2$. If the tumor burden was determined to be high, or difficulties in feeding the mice were experienced, then mice were sacrificed early. Mice were sacrificed after 4 weeks using $\mathrm{CO}_{2}$ ( $20 \%$ of the chamber volume/min) until they stopped breathing, and tumors were weighed.

Statistical analysis. Statistical analyses were performed using SPSS 19.0 (IBM Corp.). Data are presented as the mean \pm standard deviation. Between-group comparisons were conducted using single-factor analysis of variance (one-way ANOVA). Comparisons among multiple groups were performed using ANOVA followed by the least significant difference test. The in vitro results represent three independent experiments. $\mathrm{P}<0.05$ was considered to indicate a statistically significant difference.

\section{Results}

Talin1 expression is upregulated in CRC and regulates cancer cell proliferation. Talin1 protein expression levels were detected by IHC in tumor and adjacent normal tissues from each patient. Across all patients, the percent area stained for talin1 was 3.5-fold greater in tumor tissue compared with adjacent normal tissue $(\mathrm{P}<0.001$; Fig. $1 \mathrm{~A}$ and $\mathrm{B})$, suggesting that talin1 expression was upregulated in CRC. 
A

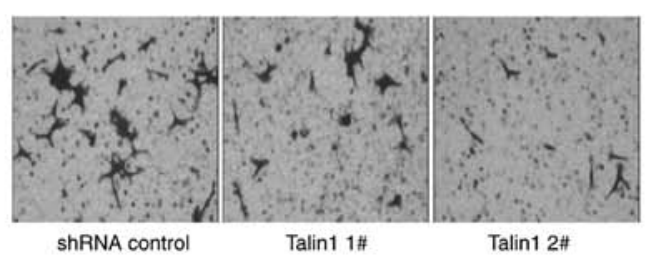

C
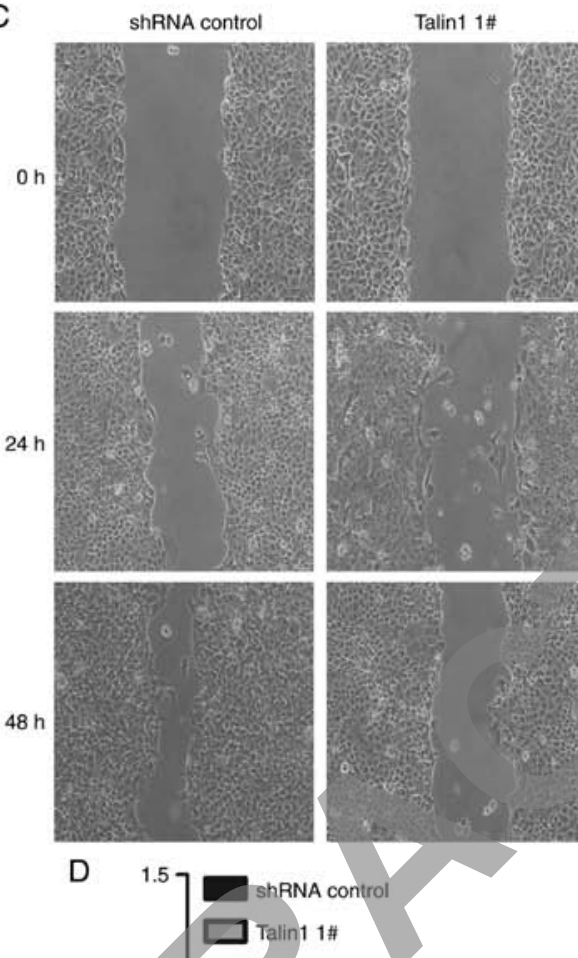

Talin1 1\#
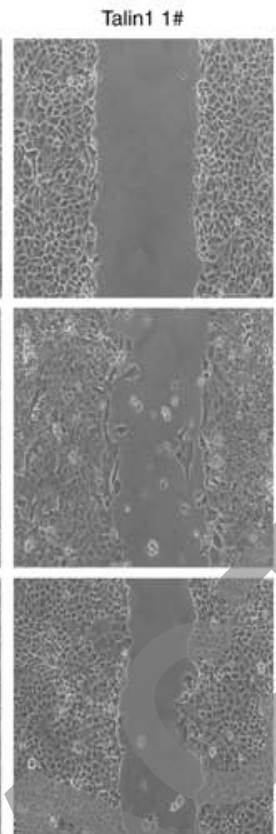
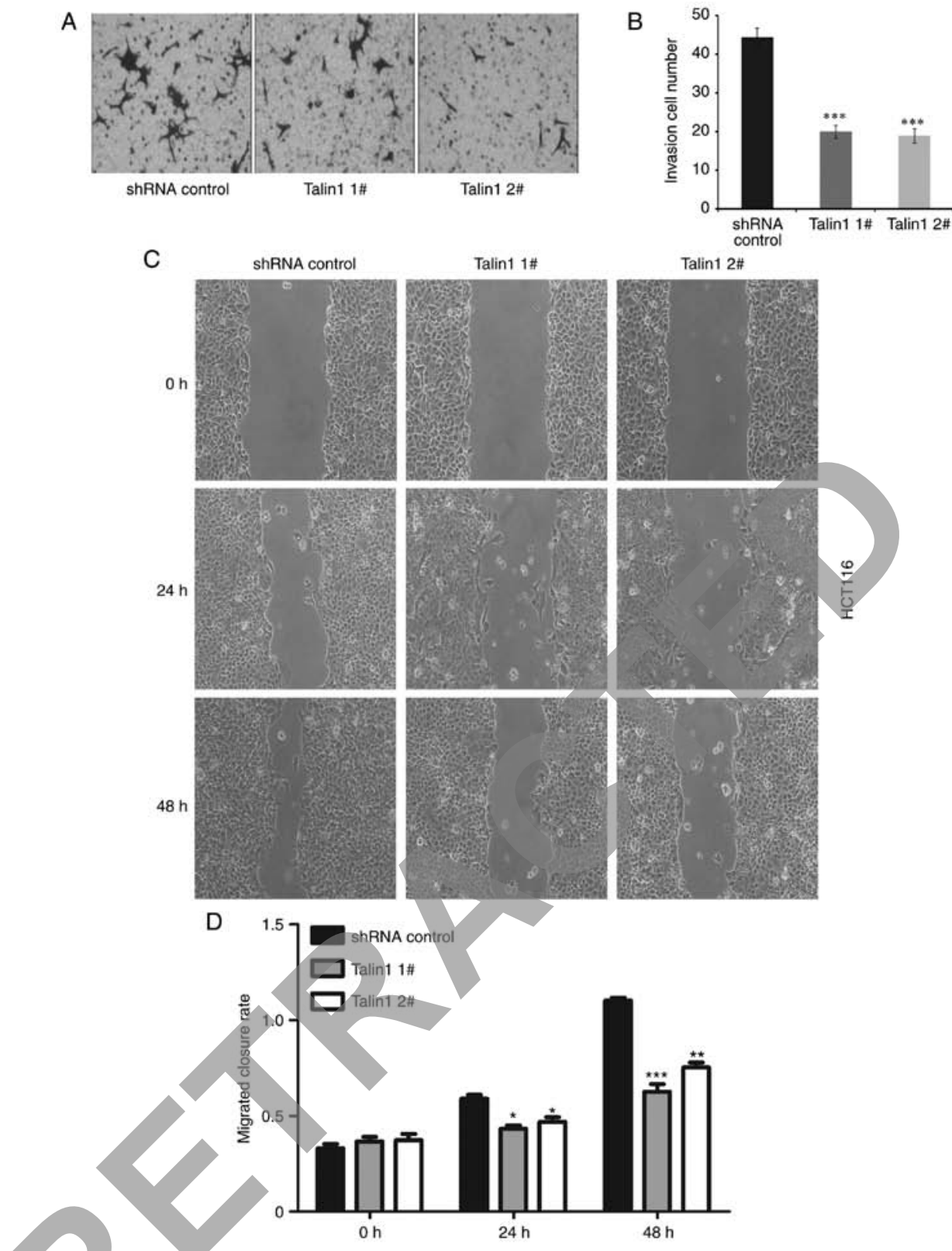

Figure 2. Talin1 knockdown inhibits the migration and invasion of HCT116 cells. (A) Knockdown of endogenous talin1 inhibited the invasive ability of HCT116 cells. (B) Quantification results of the invasion assays. (C) Talin1 knockdown reduces migration of HCT116 cells. Magnification, x400. (D) Quantification results of the wound-healing assays. Data are expressed as the mean \pm SEM from at least three independent experiments. ${ }^{*} \mathrm{P}<0.05,{ }^{* * *} \mathrm{P}<0.01$ and ${ }^{* * * *} \mathrm{P}<0.001 \mathrm{vs}$. control. shRNA, short hairpin RNA.

Stable talin1 1\#- or talin1 2\#-knockdown HCT116 cell lines were established by infecting HCT116 cells with talin1 recombinant shRNA-expressing lentiviral vectors, screening with puromycin for 3-4 weeks and subsequently allowed to grow. Relative talin 1 protein and mRNA levels were $30 \%$ lower in talin1-knockdown HCT116 cells compared with HCT116 cells transfected with shRNA control $(\mathrm{P}<0.001$; Fig. 1C-E), confirming the establishment of stable talin1-knockdown HCT116 cell lines, which were used in subsequent experiments.

Cell viability was assessed using the MTT assay. At $48 \mathrm{~h}$, cell proliferation was significantly inhibited in talin1 knockdown cell lines compared with shRNA control cells. The antiproliferative effects of talin1 knockdown were more pronounced after $24 \mathrm{~h}$ and significantly reduced after $48 \mathrm{~h}$ of culture $(\mathrm{P}<0.01$; Fig. $1 \mathrm{~F})$.
Talin1 regulates cancer cell invasion and migration. The invasive potential of talin1 1\#- or talin1 2\#-knockdown HCT116 cell lines and HCT116 cells transfected with the shRNA control was examined using Matrigel-coated Transwell chambers. Talin1-knockdown significantly reduced the invasiveness of talin1-knockdown HCT116 cells (Talin1 1\#, 55\%; Talin1 2\#, 62\%) compared with that of shRNA control cells (Fig. 2A and B).

The role of talin1 in the regulation of CRC cell migration was examined using a wound-healing assay. At $36 \mathrm{~h}$, the rate of wound closure in the talin1-knockdown cell lines was significantly reduced compared with the control $(\mathrm{P}<0.05)$. At $48 \mathrm{~h}$, the rate of wound closure in the talin1 knockdown cell lines was reduced by 0.579 -fold (talin1 $1 \#$ ) and 0.584 -fold (talin1 2\#) compared with the control (Fig. 2C and D). 
A

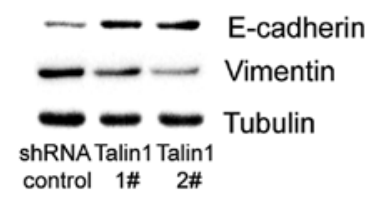

D

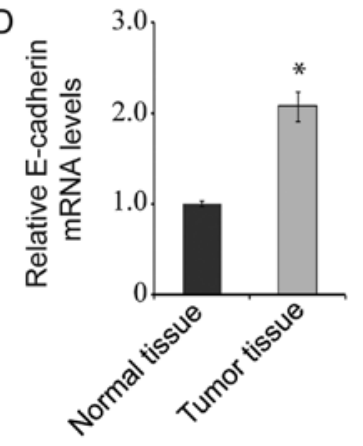

G

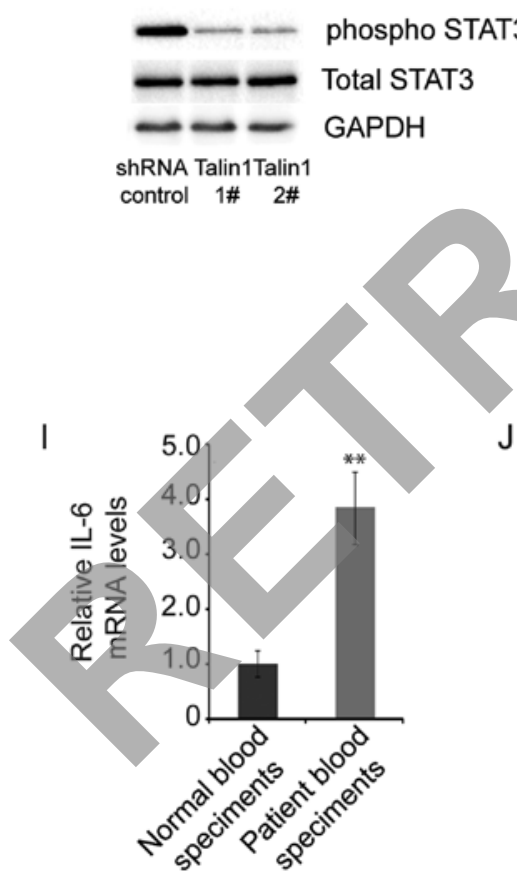

B

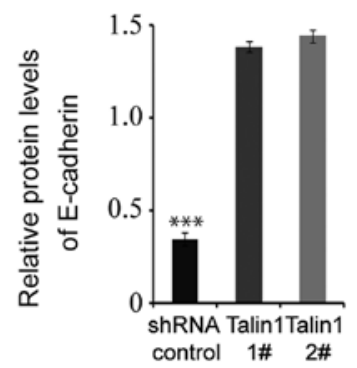

E

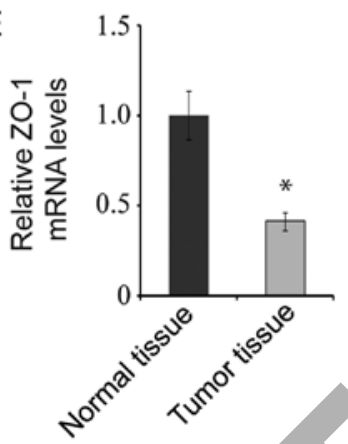

C

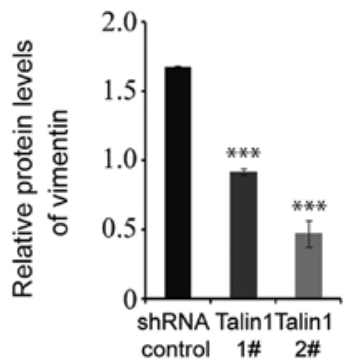

$\mathrm{F}$

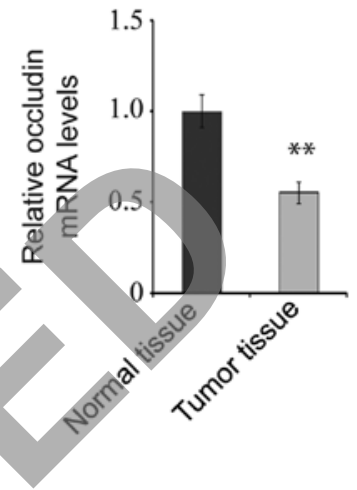

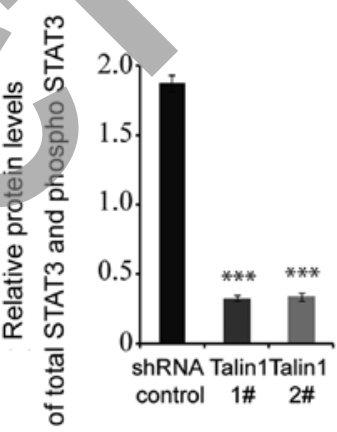
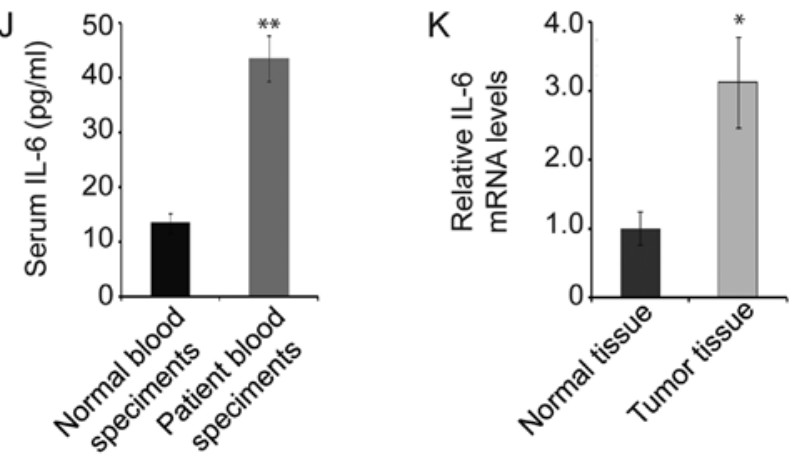

Figure 3. Talin1 knockdown affects the expression of proteins associated with the EMT signaling pathway in HCT116 cells. (A) E-cadherin and vimentin protein levels were measured by western blotting in talin1-knockdown HCT116 cell lines and HCT116 cells transfected with the shRNA control. (B) E-cadherin protein levels in talin1-knockdown HCT116 cell lines and HCT116 cells transfected with the shRNA control (n=3). (C) Vimentin protein levels in in talin1-knockdown HCT116 cell lines and HCT116 cells transfected with the shRNA control $(n=3)$. (D) E-cadherin mRNA levels in tumor and normal tissues. (E) ZO-1 mRNA levels in tumor and normal tissues. (F) Occludin mRNA levels in tumor and normal tissues. (G) pSTAT3 and total STAT3 protein levels measured by western blotting. (H) pSTAT3 and total STAT3 protein levels in talin1-knockdown HCT116 cell lines and HCT116 cells transfected with the shRNA control (n=3). (I) IL-6 mRNA levels in blood samples from patients and healthy controls. (J) Serum IL-6 concentrations in blood samples from patients and healthy controls. (K) IL-6 mRNA levels in tumor and adjacent normal tissues. Data are presented as mean \pm SEM. ${ }^{*} \mathrm{P}<0.05,{ }^{* *} \mathrm{P}<0.01$ and ${ }^{* * *} \mathrm{P}<0.001$ vs. control. ZO-1, zonula occludens-1; pSTAT3, phosphorylated STAT3; IL-6, interleukin-6; shRNA, short hairpin RNA.

Talin1 knockdown affects the expression levels of proteins associated with the EMT signaling pathway. The levels of proteins in the EMT signaling pathway in talin1 1\#- or talin1 2\#-knockdown HCT116 cell lines and HCT116 cells transfected with shRNA control were analyzed to explore the mechanism by which talin1 regulates cancer cell invasion and migration. Protein levels of phosphorylated STAT3 (pSTAT3), an oncogene that modulates cellular processes such as invasion and metastasis (34), were significantly lower in talin1-knockdown HCT116 cell lines compared with the control (Fig. 3G and H). 
A

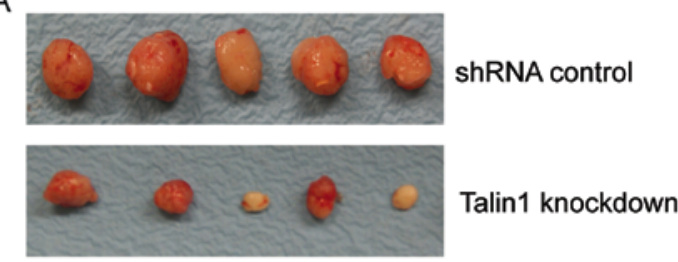

C

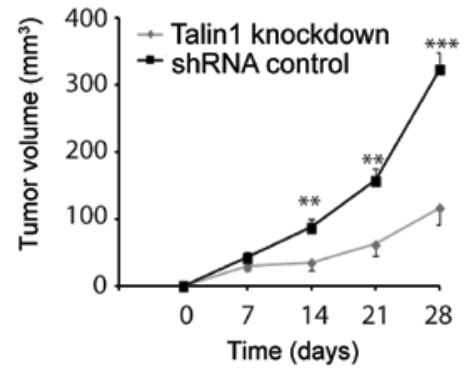

B

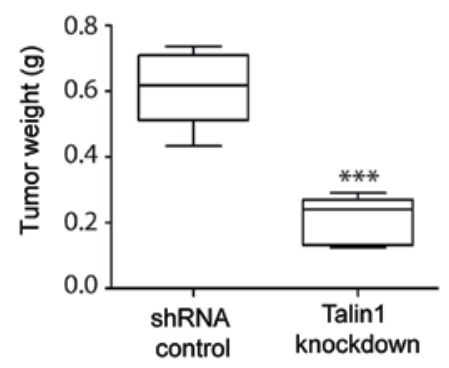

D

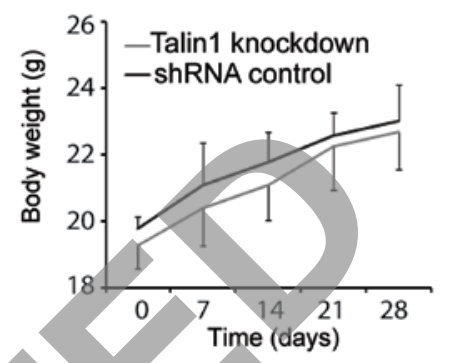

Figure 4. Talin1 knockdown suppressed tumor growth in a xenograft model. (A) Talin1 knockdown inhibited tumor growth. (B and C) Talin1 knockdown reduced (B) tumor weight and (C) tumor volume. (D) Talin1 knockdown did not affect mouse body weight. " $\mathrm{P}<0.01$ and $" \mathrm{P}<0.001$ vs. control. shRNA, short hairpin RNA.

Protein levels of vimentin, a marker of EMT, were also significantly lower in talin1-knockdown HCT116 cell lines compared with the control, whereas mRNA and protein levels of E-cadherin were increased (Fig. 3A-D). These results suggested that talin1 knockdown inhibits CRC tumor invasion through the EMT signaling pathway.

qPCR indicated that mRNA levels of ZO-1 and occludin, which are major components of tight junctions, decreased significantly in tumor tissue compared with adjacent normal tissue (Fig. 3E-F). As a major activator of STAT3 (35), mRNA levels of IL-6 were detected in blood, tumor and adjacent normal tissue samples from each patient. IL- 6 mRNA levels were significantly increased in patient blood samples compared with blood samples from healthy controls, as well as in tumor tissue compared with adjacent normal tissue (Fig. 3I and J). ELISA results demonstrated that levels of IL-6 in patient blood samples were 3.2-fold higher compared with those in healthy individuals (Fig. 3K).

Talin1 knockdown suppresses tumor growth. To evaluate the potential of talin1 as a therapeutic target, the effect of talin1 knockdown on the growth of tumor xenografts in BALB/c nude mice was examined. Mice were injected subcutaneously with talin1 1\#- or talin1 2\#-knockdown HCT116 cell lines or HCT116 cells transfected with the shRNA control. Talin1 knockdown inhibited tumor growth, resulting in significantly smaller tumors with reduced volumes compared to control (Fig. 4A and C). Talin1 knockdown decreased tumor mass by 63.81\% compared with the control (Fig. 4B), but did not affect mouse body weight (Fig. 4D). These data suggested that talin 1 knockdown may inhibit tumor growth in vivo.

\section{Discussion}

The present study determined talin1 expression levels in CRC and investigated the role of talin1 in CRC proliferation and invasion in vitro and in vivo. IHC analysis of human CRC and adjacent normal tissue demonstrated that talin1 expression was upregulated in CRC. Talin1 knockdown significantly reduced the proliferative, migratory and invasive abilities of HCT116 cells compared with the control. Talin1 knockdown affected the expression of proteins in the EMT signaling pathway in HCT116 cells. qPCR and ELISA results also showed that IL-6 levels were significantly increased in blood samples obtained from patients with tumors compared with the control. In vivo experiments revealed that talin1 knockdown reduced CRC tumor growth and weight in nude mice. These results show that Talin1 knockdown decreases proliferation and migration of CRC cells by downregulating various factors involved in EMT.

Talin1 is a ubiquitous cytoskeleton-associated protein that couples the cytoskeleton with integrins and is an essential mediator of cell-ECM adhesion. The N-terminal head domain of talin1 binds to the cytoplasmic domain of $\beta$ integrin, which causes integrin activation and stimulates integrin binding to the ECM $(36,37)$. Integrin adhesion to the ECM can be further modified, as talin1 links the ECM and actin by binding to actin filaments, and actin and/or integrins unfold talin1 domains, which results in the exposure of binding sites for additional integrin-associated proteins, such as vinculin and FAK $(9,38)$. Talin1 can also act independently of integrins, regulating the expression of E-cadherin, which is a cell-cell adhesion molecule associated with impaired cancer progression (10). Talin1 has been identified to serve important roles in various types of disease (38-41). Specifically, Xu et al (32) demonstrated that upregulated talin-1 expression was associated with malignant behavior in prostate cancer and lymph node metastasis. Upregulation of talin1 has also been demonstrated in hepatocellular carcinoma progression and may serve as a prognostic marker (42). In the present study, Talin1 protein expression levels were determined to be upregulated in tumor tissues compared with the adjacent normal tissues. 
STAT3 is an oncogene activated by phosphorylation of a conserved tyrosine residue in response to the binding of cytokines, hormones and growth factors to their receptors and activation of Janus kinase 2 or the intrinsic tyrosine kinase activity of the receptors (16). Phosphorylation of STAT3 promotes its homodimerization and translocation to the nucleus, where it regulates the transcription of target genes that modulate essential cellular processes, such as proliferation and differentiation (16). The results of the present study demonstrated that the levels of pSTAT3 were decreased in talin1-knockdown HCT116 cell lines, suggesting that talin1 knockdown inhibits CRC tumor invasion.

EMT involves dissolution of epithelial cell-cell junctions, establishment of a front-rear polarity, reorganization of the cytoskeleton, changes in cell shape and increased cell motility and invasive behavior (43). EMT is accompanied by decreased E-cadherin levels during destabilization of adherens junctions and increased vimentin levels; vimentin interacts with motor proteins and facilitates cell motility (43-46). In the present study, E-cadherin expression was increased and vimentin expression was decreased in talin1-knockdown HCT116 cell lines compared with the control. These findings suggested that talin 1 knockdown inhibited cell invasive capability, possibly through the EMT signaling pathway.

Persistent infections and chronic inflammation may be a causative factor in certain types of cancer, such as colitis-associated cancer, hepatocellular carcinoma and skin cancer, and a number of tumors exhibit extensive immune cell infiltration with high levels of inflammatory mediators in the tumor microenvironment $(22,47,48)$. In CRC, serum levels of IL- 6 have been reported to be the highest in patients with advanced clinical stage or metastatic disease (49). Consistent with this, the qPCR results in the present study demonstrated that IL-6 levels were higher in human CRC tumors compared with adjacent normal tissue and the ELISA results showed that the levels of IL-6 in patient blood samples were 3.2-fold higher compared with those in healthy individuals.

In conclusion, the present study demonstrated that talin1 was upregulated in human $\mathrm{CRC}$ and that talin1 knockdown prevented the proliferation and migration of CRC cells via the EMT signaling pathway. EMT associated with cancer progression represents a potential treatment strategy in clinical oncology; therefore, talin 1 may provide a novel therapeutic target for CRC. Future studies are to focus on the generation of a conditional knockout mouse model of talin1, selectively targeting talin1 gene expression in colorectal cells, crossed with a transgenic mouse model of colorectal cancer.

\section{Acknowledgements}

Not applicable.

\section{Funding}

The present study was funded by the Shandong Key Research and Development Plan (grant nos. 2017GSF218035 and 2013G0021810).

\section{Availability of data and materials}

The datasets used and/or analyzed during the present study are available from the corresponding author on reasonable request.

\section{Authors' contributions}

LJ and CQ conceived and designed the experiments. LJ and $\mathrm{XC}$ performed the experiments. LJ, FJ, XC and CQ analyzed the data. FJ contributed the materials and analysis tools. LJ and CQ wrote the manuscript. All authors read and approved the final manuscript.

\section{Ethical approval and consent to participate}

This study was approved by the Board and Ethical Committee of The First Affiliated Hospital of Wenzhou Medical University. Written informed consent in accordance with the Declaration of Helsinki was obtained from all study subjects. Animal experiments were performed in accordance with the Guide for the Care and Use of Laboratory Animals. All animal experimental procedures were approved by the Wenzhou Medical University Animal Policy and Welfare Committee.

\section{Patient consent for publication}

All patients provided written informed consent for the publication of their data.

\section{Competing interests}

The authors declare that they have no competing interests.

\section{References}

1. Pattison AM, Merlino DJ, Blomain ES and Waldman SA: Guanylyl cyclase $\mathrm{C}$ signaling axis and colon cancer prevention. World J Gastroenterol 22: 8070-8077, 2016.

2. Torre LA, Bray F, Siegel RL, Ferlay J, Lortet-Tieulent J and Jemal A: Global cancer statistics, 2012. CA Cancer J Clin 65: 87-108, 2015

3. Arnold M, Sierra MS, Laversanne M, Soerjomataram I, Jemal A and Bray F: Global patterns and trends in colorectal cancer incidence and mortality. Gut 66: 683-691, 2017.

4. Frampton M and Houlston RS: Modeling the prevention of colorectal cancer from the combined impact of host and behavioral risk factors. Genet Med 19: 314-321, 2017.

5. Das M, Ithychanda S, Qin J and Plow EF: Mechanisms of talin-dependent integrin signaling and crosstalk. Biochim Biophys Acta 1838: 579-588, 2014.

6. Calderwood DA and Ginsberg MH: Talin forges the links between integrins and actin. Nat Cell Biol 5: 694-697, 2003.

7. Hu X, Jing C, Xu X, Nakazawa N, Cornish VW, Margadant FM and Sheetz MP: Cooperative vinculin binding to talin mapped by time-resolved super resolution microscopy. Nano Lett 16 : 4062-4068, 2016.

8. Giancotti FG and Ruoslahti E: Integrin signaling. Science 285: 1028-1032, 1999.

9. Klapholz B and Brown NH: Talin-the master of integrin adhesions. J Cell Sci 130: 2435-2446, 2017.

10. Becam IE, Tanentzapf G, Lepesant JA, Brown NH and Huynh JR: Integrin-independent repression of cadherin transcription by talin during axis formation in Drosophila. Nat Cell Biol 7: 510-516, 2005

11. Zhang JL, Qian YB, Zhu LX and Xiong QR: Talin1, a valuable marker for diagnosis and prognostic assessment of human hepatocelluar carcinomas. Asian Pac J Cancer Prev 12: 3265-3269, 2011.

12. Sakamoto $S$ and Kyprianou N: Targeting anoikis resistance in prostate cancer metastasis. Mol Aspects Med 31: 205-214, 2010. 
13. Lai MT, Hua CH, Tsai MH, Wan L, Lin YJ, Chen CM, Chiu IW, Chan C, Tsai FJ and Jinn-Chyuan Sheu J: Talin-1 overexpression defines high risk for aggressive oral squamous cell carcinoma and promotes cancer metastasis. J Pathol 224: 367-376, 2011.

14. Chen P, Zheng X, Zhou Y, Xu Y, Zhu L and Qian Y: Talin-1 interaction network promotes hepatocellular carcinoma progression. J Cell Sci 8: 13003-13014, 2017.

15. Kalluri R and Weinberg RA: The basics of epithelial-mesenchymal transition. J Clin Invest 119: 1420-1428, 2009.

16. Huang C, Yang G, Jiang T, Zhu G, Li H and Qiu Z: The effects and mechanisms of blockage of STAT3 signaling pathway on IL-6 inducing EMT in human pancreatic cancer cells in vitro. Neoplasma 58: 396-405, 2011.

17. Chang KC, Wu MH, Jones D, Chen FF and Tseng YL: Activation of STAT3 in thymic epithelial tumours correlates with tumour type and clinical behaviour. J Pathol 210: 224-233, 2006.

18. Kusaba T, Nakayama T, Yamazumi K, Yakata Y, Yoshizaki A, Nagayasu T and Sekine I: Expression of p-STAT3 in human colorectal adenocarcinoma and adenoma; correlation with clinicopathological factors. J Clin Pathol 58: 833-838, 2005.

19. Grandis JR, Drenning S, Zeng Q, Watkins SC, Melhem MF, Endo S, Johnson DE, Huang L, He Y and Kim JD: Constitutive activation of Stat 3 signaling abrogates apoptosis in squamous cell carcinogenesis in vivo. Proc Natl Acad Sci USA 97: 4227-4232, 2000.

20. Dhir R, Ni Z, Lou W, DeMiguel F, Grandis JR and Gao AC: Stat 3 activation in prostatic carcinomas. Prostate 51: 241-246, 2002

21. Scholz A, Heinze S, Detjen KM, Peters M, Welzel M, Hauff P, Schirner M, Wiedenmann B and Rosewicz S: Activated signal transducer and activator of transcription 3 (STAT3) supports the malignant phenotype of human pancreatic cancer. Gastroenterology 125: 891-905, 2003.

22. Grivennikov SI, Greten FR and Karin M: Immunity, inflammation and cancer. Cell 140: 883-899, 2010.

23. Hanahan D and Weinberg RA: Hallmarks of cancer: The next generation. Cell 144: 646-674, 2011.

24. Garbers C, Hermanns HM, Schaper F, Muller-Newen G Grotzinger J, Rose-John S and Scheller J: Plasticity and cross-talk of interleukin 6-type cytokines. Cytokine Growth Factor Rev 23. 85-97, 2012.

25. Middleton K, Jones J, Lwin Z and Coward JI: Interleukin-6: An angiogenic target in solid tumours. Crit Rev Oncol Hematol 89: $129-139,2014$

26. Xu Q, Briggs J, Park S, Niu G, Kortylewski M, Zhang S, Gritsko T, Turkson J, Kay H, Semenza G, et al: Targeting Stat 3 blocks both HIF-1 and VEGF expression induced by multiple oncogenic growth signaling pathways. Oncogene 24: 5552-5560, 2005.

27. Jung JE, Lee HG, Cho IH, Chung DH, Yoon SH, Yang YM, Lee JW, Choi S, Park JW, Ye SK and Chung MH: STAT3 is a potential modulator of HIF-1-mediated VEGF expression in human renal carcinoma cells. FASEB J 19: 1296-1298, 2005

28. Paleri V, Mehanna H and Wight RG: TNM classification of malignant tumours 7th edition: What's new for head and neck? Clin Otolaryngol 35: 270-272, 2010.

29. Mosmann T: Rapid colorimetric assay for cellular growth and survival: Application to proliferation and cytotoxicity assays. J Immunol Methods 65: 55-63, 1983.

30. Wu Z, Li X, Sunkara M, Spearman H, Morris AJ and Huang C: PIPKI $\gamma$ regulates focal adhesion dynamics and colon cancer cell invasion. PLoS One 6: e24775, 2011.

31. Liu B, Pang B, Hou X, Fan H, Liang N, Zheng S, Feng B, Liu W, Guo H, Xu S and Pang Q: Expression of high-mobility group AT-hook protein 2 and its prognostic significance in malignant gliomas. Hum Pathol 45: 1752-1758, 2014.

32. Xu N, Chen HJ, Chen SH, Xue XY, Chen H, Zheng QS, Wei Y, Li XD, Huang JB, Cai H and Sun XL: Upregulation of Talin-1 expression associates with advanced pathological features and predicts lymph node metastases and biochemical recurrence of prostate cancer. Medicine 95: e4326, 2016.
33. Livak KJ and Schmittgen TD: Analysis of relative gene expression data using real-time quantitative PCR and the 2(-Delta Delta C(T)) method. Methods 25: 402-408, 2001.

34. Avalle L, Camporeale A, Camperi A and Poli V: STAT3 in cancer: A double edged sword. Cytokine 98: 42-50, 2017.

35. Hodge DR, Hurt EM and Farrar WL: The role of IL-6 and STAT3 in inflammation and cancer. Eur J Cancer 41: 2502-2512, 2005.

36. Critchley DR: Biochemical and structural properties of the integrin-associated cytoskeletal protein talin. Annu Rev Biophys 38: 235-254, 2009.

37. Anthis NJ, Wegener KL, Ye F, Kim C, Goult BT, Lowe ED, Vakonakis I, Bate N, Critchley DR, Ginsberg MH and Campbell ID: The structure of an integrin/talin complex reveals the basis of inside-out signal transduction. EMBO J 28 3623-3632, 2009

38. Kang W, Kim SH, Cho HJ, Jin J, Lee J, Joo KM and Nam DH: Talin1 targeting potentiates anti-angiogenic therapy by attenuating invasion and stem-like features of glioblastoma multiforme. Oncotarget 6: 27239-27251, 2015.

39. Muto M, Mori M, Hiwasa T, Takiguchi M, Iwadate Y, Uzawa A, Uchida T, Masuda H, Sugimoto K and Kuwabara S: Novel serum autoantibodies against talin 1 in multiple sclerosis: Possible pathogenetic roles of the antibodies. J Neuroimmunol 284 30-36, 2015.

40. Singel SM, Cornelius C, Batten K, Fasciani G, Wright WE, Lum L and Shay JW: A targeted RNAi screen of the breast cancer genome identifies KIF14 and TLN1 as genes that modulate docetaxel chemosensitivity in triple-negative breast cancer. Clin Cancer Res 19: 2061-2070, 2013.

41. Sen S, Ng WP and Kumar S: Contributions of talin-1 to glioma cell-matrix tensional homeostasis. J R Soc Interface 9: 1311-1317, 2012.

42. Kanamori H, Kawakami T, Effendi K, Yamazaki K, Mori T, Ebinuma H, Masugi Y, Du W, Nagasaka K, Ogiwara A, et al: Identification by differential tissue proteome analysis of talin-1 as a novel molecular marker of progression of hepatocellular carcinoma. Oncology 80: 406-415, 2011.

3. Lamouille S, Xu J and Derynck R: Molecular mechanisms of epithelial-mesenchymal transition. Nat Rev Mol Cell Biol 15: 178-196, 2014.

44. Thiery JP: Epithelial-mesenchymal transitions in tumour progression. Nat Rev Cancer 2: 442-454, 2002

45. Kang Y and Massague J: Epithelial-mesenchymal transitions: Twist in development and metastasis. Cell 118: 277-279, 2004.

46. Onder TT, Gupta PB, Mani SA, Yang J, Lander ES and Weinberg RA: Loss of E-cadherin promotes metastasis via multiple downstream transcriptional pathways. Cancer Res 68: 3645-3654, 2008.

47. Grivennikov SI and Karin M: Inflammatory cytokines in cancer: Tumour necrosis factor and interleukin 6 take the stage. Ann Rheum Dis 70 (Suppl 1): i104-i108, 2011.

48. Elinav E, Nowarski R, Thaiss CA, Hu B, Jin C and Flavell RA: Inflammation-induced cancer: Crosstalk between tumours, immune cells and microorganisms. Nat Rev Cancer 13: 759-771, 2013.

49. Eldesoky A, Shouma A, Mosaad Y and Elhawary A: Clinical relevance of serum vascular endothelial growth factor and interleukin-6 in patients with colorectal cancer. Saudi J Gastroenterol 17: 170-173, 2011.

This work is licensed under a Creative Commons Attribution-NonCommercial-NoDerivatives 4.0 International (CC BY-NC-ND 4.0) License. 